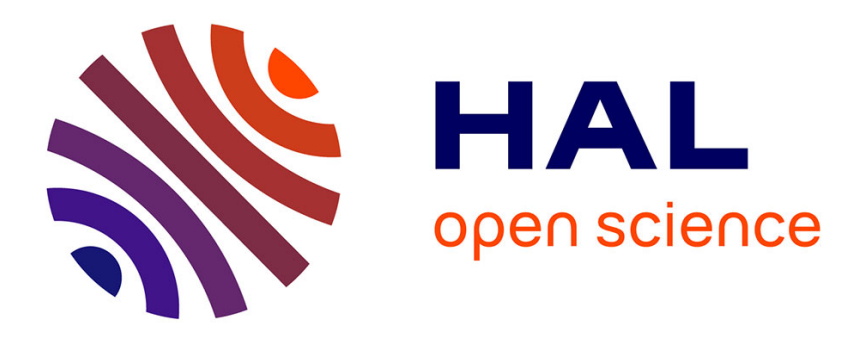

\title{
Sous la légende. Le récit inédit du renseignement français
}

Eve Lamendour

\section{To cite this version:}

Eve Lamendour. Sous la légende. Le récit inédit du renseignement français. Hermès, La Revue - Cognition, communication, politique, 2016, Le renseignement, un monde fermé dans une société ouverte, 76 (3), http://www.cairn.info.gutenberg.univ-lr.fr/revue-hermes-la-revue-2016-3.htm. 10.3917/herm.076.0162 . hal-01639578

\section{HAL Id: hal-01639578 \\ https: / hal-univ-rochelle.archives-ouvertes.fr/hal-01639578}

Submitted on 20 Nov 2017

HAL is a multi-disciplinary open access archive for the deposit and dissemination of scientific research documents, whether they are published or not. The documents may come from teaching and research institutions in France or abroad, or from public or private research centers.
L'archive ouverte pluridisciplinaire $\mathbf{H A L}$, est destinée au dépôt et à la diffusion de documents scientifiques de niveau recherche, publiés ou non, émanant des établissements d'enseignement et de recherche français ou étrangers, des laboratoires publics ou privés. 
H76 - 0**_Lamendour_Sous la légende

Ève Lamendour

Institut d'administration des entreprises - université de La Rochelle

\section{Sous la légende. Le récit inédit du renseignement français}

Le monde du renseignement dépeint par la culture populaire est celui de l'espionnage anglo-saxon. Si l'on s'intéresse au roman, le constat est patent et permettrait de lister un nombre impressionnant d'auteurs prolifiques : parmi ceux-ci, citons l'Américain Robert Ludlum notamment pour la série construite autour du diplomate vengeur Jason Bourne, démarrée en 1980 et poursuivie après le décès de Ludlum, les Britanniques Graham Greene (L'Agent secret, $1939^{1}$ ) et John Le Carré (L'Espion qui venait du froid, 1963, ou La Taupe, 1974). Parmi les textes originels, il faut citer L'Agent secret (1907) de Joseph Conrad, un roman adapté au cinéma et à la télévision à de multiples occasions, et le premier bestseller de l'Américain James Fenimore Cooper, L'Espion (1821). Au cinéma, les films d'espionnage abondent. Les plus populaires enchaînent de véritables séries autour de leur héros, à l'image du très Britannique James Bond (24 films depuis 1962) ou de l'Américain Jason Bourne (4 films depuis 2002). Quant à la télévision, elle offre, par le jeu des diffusions et rediffusions et par la multiplication des canaux de diffusion (de l'internet au dvd), une accumulation de séries anglaises ou américaines : Chapeau melon et bottes de cuir (1961-1969), Le Prisonnier (1967-1968), 24 heures chrono (2001-2014) ou Homeland (2011-2015). Si des auvres françaises existent, elles n'ont pas atteint la notoriété de la production anglo-saxonne. Ces créations françaises sont pour partie des pastiches de la production anglophone, de MarieChantal contre Dr Kha réalisé par Claude Chabrol en 1965 aux différentes versions cinématographiques d'OSS 117. Pourtant, une série télévisée récente, Le Bureau des légendes, marque un récit inédit dans ce qui serait une représentation typiquement française de l'activité de renseignement. S'en saisir en comparant ce récit avec les procédés propres aux représentations anglo-saxonnes va permettre de détailler les spécificités françaises, et surtout de comprendre la nature des résistances à intégrer le renseignement dans l'imaginaire national.

\section{Un imaginaire indemne du fantasme de l'espion}

En examinant la profusion de la production télévisuelle anglophone en matière d'espionnage, le désintérêt de la création française pour ce sujet apparaît plus marquant. Le détour par la fiction étrangère va permettre d'éclairer cette situation.

\section{Une préoccupation d'essence nationaliste}

Si l'on doit faire un bref état des lieux de la production télévisée de fiction consacrée à l'espionnage, deux aspects sont frappants : la place prédominante du monde anglo-saxon dans la représentation et l'ethnocentrisme du propos. Avant d'aborder le premier point, remarquons que les fictions nationales se concentrent massivement sur les services secrets de leur Etat d'origine, qu'il s'agisse de sécurité intérieure ou extérieure. Les agences mises en scène sont alors parfois explicitement le Federal Bureau of Investigation (FBI) pour les Etats-Unis à travers par exemple la série Duo très spécial (six saisons démarrées en 2009) ou le MI5 pour

\footnotetext{
${ }^{1}$ Par souci de lisibilité, les titres des ouvrages, films et séries télévisées figurent en français suivi de la date de publication ou diffusion originale.
} 
la Grande-Bretagne vu à travers Spooks, série créée en 2002 et qui, en dix saisons, a bénéficié d'une large reconnaissance avec trente-cinq nominations et dix récompenses majeures. La référence à une agence de renseignement précise peut ne pas être explicite sans pour autant manquer des marqueurs nationaux : la série Le Prisonnier tournée en grande partie au Pays de Galles présente tous les éléments de typicité britannique, de Big Ben à la voiture de l'agent démissionnaire, une Lotus Seven. Le fait de traiter de la sécurité extérieure n'apporte guère aux séries un point de vue plus ouvert. Les agences alors mises en scène sont toujours des agences nationales, respectivement la Central Intelligence Agency (CIA) dans les séries produites aux Etats-Unis (Homeland - six saisons, 2011), le MI6 (London Spy - 2015) en Grande-Bretagne, la HVA, agence de l'ex-République démocratique allemande (RDA), en Allemagne (Deutschland 83 - 2015), ou la direction générale de la Sécurité extérieure (DGSE) en France (Le Bureau des légendes - deux saisons, 2015). La représentation de la menace extérieure ou simplement du monde étranger est proche du stéréotype, voire de la caricature : Beyrouth en camp retranché dans Homeland a ainsi fait réagir les réseaux sociaux, les artistes et le gouvernement libanais ${ }^{2}$.

\section{Un prisme anglo-saxon}

Pour ce qui est de la prédominance d'une représentation anglo-saxonne, il convient de nuancer la proposition. D'une part, l'axiome est plus juste pour ce qui concerne le cinéma que pour la littérature. La série de romans consacrés à OSS 117, agent américain, qui a inspiré les films éponymes a été initiée en 1949 par l'auteur français Jean Bruce et compte 255 titres. Aussi prolifique, la série SAS due à la plume de Gérard de Villiers cumule 200 titres publiés à partir de 1965. Parmi les devanciers de cette veine populaire à large diffusion, on peut remarquer une forme de romans d'espionnage à teneur nationaliste dont Les Cinq Cents Millions de la Bégum serait représentatif. Publié par Jules Verne en 1879, ce roman d'espionnage lié au conflit franco-prussien sera actualisé en 1919 dans une adaptation cinématographique. D'autre part, il n'y a pas une représentation univoque qui serait propre au monde anglophone. En se concentrant sur les seules séries télévisées, on voit dans la création américaine des productions pléthoriques couvrant tous les genres, du comique (Max la menace - 1965-1970; Chuck - 2007-2012) au récit initiatique particulièrement bien représenté (Alias - 2001-2006 ; Dollhouse - 2009-2010 ; Quantico - 2015-, etc.), toutes les époques, du XVIII siècle (Turn - 2014-) à l'époque contemporaine (Burn notice - 2007-2013) en passant par le XIX siècle (Les Mystères de l'Ouest - 1965-1969) ou les années 1940 (Agent Carter - 2015-2016). La télévision britannique offre aussi une production nombreuse où émergent deux tonalités peu perceptibles dans les séries d'outre-Atlantique : l'humour et un goût pour l'absurde. Une série a su mêler ces deux aspects : Chapeau melon et bottes de cuir (1961-1969).

Si l'on devait formuler une spécificité à ces œuvres, on pourrait dire que le maitre-mot des séries étatsuniennes est l'idée que l'ennemi est parmi nous. Il est caché, se fait passer pour Américain et il convient de le dévoiler. La série ironiquement intitulée The Americans (2013-) propose une incarnation de cette idée. Elle montre deux espions soviétiques infiltrés aux Etats-Unis au début des années 1980 former un couple, avoir des enfants et mener une vie de banlieusards des plus anodines tout en exerçant une activité de déstabilisation de l'Etat reaganien en faveur d'un gouvernement ennemi. La notion d'une contamination qu'il convient de circonvenir, d'une pureté à retrouver en démasquant l'ennemi infiltré, est de fait

\footnotetext{
${ }^{2}$ Pour apprécier l'ampleur des réactions, voir <www.france24.com/fr/20151015-homeland-serie-raciste-artistestags-production-serie-americaine-monde-arabe-islam $>$ et $<$ www.france24.com/fr/20121102-homeland-ustelevision-showtime-liban-claire-danes-beyrouth-beirut-back-hamra> (pages consultées le 10/09/2016).
} 
fondatrice dans le récit propre aux Etats-Unis - que l'on pense à La Lettre écarlate, le roman de Hawthorne où il est question d'une littérale chasse aux sorcières (1850) ou aux réelles persécutions à visée purificatrice du maccarthysme dont les scénaristes hollywoodiens ont été en particulier victimes aux débuts des années 1950. On le voit, cet ennemi à démasquer au sein même de la société dépasse le champ du récit d'espionnage, et cette peur contamine la fiction étatsunienne à travers de nombreux genres. A l'écran, les vampires de True blood (2008-2014) tiennent à la perfection ce rôle de voisins à l'apparence bénigne partageant les habitudes de la communauté où ils se sont insérés mais dont ils ne sont irréductiblement pas membres de droit.

La fiction anglaise d'espionnage repose davantage sur l'idée que nous sommes l'ennemi. L'ennemi n'est pas représenté comme un étranger masqué mais bien comme l'un de nous passé à l'ennemi. La cause est-elle à trouver dans l'histoire contemporaine et l'affaire des cinq de Cambridge qui occupèrent des postes importants au sein de la diplomatie et des services de renseignement anglais tout en œuvrant pour l'Union soviétique ? La paranoïa centrée sur la recherche de l'ennemi intérieur est au cœur du récit du Prisonnier, de Hunted (2012), The Game (2014), London Spy (2015), est sensible au fil des saisons de Spooks, est aussi présente dans The Hour (2011-2012). La focale est placée sur le dérèglement d'institutions de renseignement en charge de protéger les personnes sur le territoire national.

\section{Des jeux d'influence sur un pays dépourvu d'un imaginaire du renseignement}

Si les fictions nationales peuvent être typées, il n'en existe pas moins des jeux d'influence insensibles aux frontières du fait des adaptations (Homeland est ainsi adaptée de la série israélienne Hatufim - 2010-2012, et illustre une paranoïa aggravée) et du fait de la diffusion que connaissent les séries anglophones à l'étranger. La France est particulièrement concernée par ce constat produisant globalement beaucoup moins de séries que les autres pays européens et diffusant un fort volume de fictions étatsuniennes : " la fiction étrangère représente $60 \%$ des fictions diffusées en première partie de soirée en France alors que les 10 meilleures audiences de fiction correspondent à des fictions nationales en Angleterre, en Allemagne, en Espagne et en Italie, 7 des 10 meilleures audiences de fiction en France sont américaines. » (Plancade, 2013, p.34) Dans le paysage contemporain, les barbouzes du SAS font figure de sympathiques anachronismes absents du petit écran à l'instar d'OSS 117. Rare production télévisuelle française, Schulmeister, l'espion de l'Empereur (1971-1974) n'a pas eu de continuateur. Et Franck Bulinge (2012) notait à ce propos « une disparition progressive de la culture typiquement française au profit d'une culture hybride, américanisée. » Le spectateur français contemporain a une représentation du monde du renseignement principalement issue des séries américaines, soit des récits d'actions échevelées. Dans ce contexte, il est une série sans précédent, Le bureau des légendes, conçue par un réalisateur récidiviste. Eric Rochant est en effet l'auteur d'un film d'espionnage, Les Patriotes, présenté au festival de Cannes en 1994. La série, diffusée en 2015, connaît une seconde saison en 2016 et, chose rare, se vend à l'étranger (Les Echos, 7/9/2016).

\section{Avancer masqué}

\section{La paranoïa, moteur des intrigues de séries d'espionnage}

L'essence du récit d'espionnage est la dissimulation : «sous chaque personnage, si ordinaire soit-il, peut se dissimuler une autre personne [...] dont l'apparence sociale est 
d'autant plus typée qu'elle n'est qu'un déguisement destiné à tromper à la fois les autres personnages et le lecteur. » (Boltanski, 2012) Ces jeux de rôle interdisent toute certitude et toute réalité stable. L'agence de renseignement, et au-delà l'Etat dans lequel elle s'insère et toute la société, sont soumis à la suspicion (Bonin, 2007). La peur de l'autre dans la version étatsunienne (l'ennemi est caché parmi nous), la crainte de la taupe dans la production anglaise (nous sommes l'ennemi) alimentent la paranoïa. Car il s'agit bien de cette pathologie qui amène le sujet à "prolonger l'enquête au-delà du raisonnable » selon l'une des caractéristiques formulées par le psychiatre Emil Kraepelin qui, le premier, a identifié cette maladie (Boltanski, 2012). Le fantasme d'une pureté originelle n'est pas visible dans la série française ; y domine davantage une forme de précaution exacerbée vis-à-vis du système. $\mathrm{Au}$ sein de la DGSE, le service concerné est identifié comme le Bureau des légendes en charge des clandestins infiltrés à l'étranger sous une fausse identité afin de recueillir des informations et de repérer des informateurs potentiels. A la manière d'une poupée russe (l'image est fréquemment utilisée dans les génériques de films et séries d'espionnage), le personnage principal est multiple ; il est Guillaume Debailly, agent fraîchement revenu de mission en Syrie, il est aussi Malotru, son pseudonyme pour les autres membres du service, et il est Paul Lefebvre, professeur de littérature française à Damas. Le récit démarre à son retour à Paris qui signe la fin définitive de sa mission au Moyen-Orient et l'abandon de son identité d'emprunt, sa «légende » pour reprendre le vocabulaire de la série. Jouant vis-à-vis de ses collègues de prétextes divers, accumulant étourderies jouées et omissions volontaires, contre toutes les règles explicites, le personnage maintient ses différentes identités comme autant de panoplies parmi lesquelles choisir selon les circonstances. Ainsi il incarne le cloisonnement qui est habituellement décrit comme la marque des organisations secrètes (Grey, 2014). Sa résistance à ne pas redevenir un, à conserver les masques, semble lui garantir une position de supériorité par rapport à sa hiérarchie, lui permet de conserver une marge de manœuvre au sein d'une bureaucratie tatillonne et de préserver ses intérêts personnels.

\section{Une narration débarrassée du fantasme de l'espion}

Le propre de la série française est de relever d'une approche réaliste débarrassé du fatras de testostérone des versions nord-américaines. Le suspense, s'il en est, ne tient pas sur des faits d'armes et des actions violentes. L'unique action d'éclat est repoussée aux confins du récit. L'assaut d'un convoi ennemi au Sahara a lieu à la toute fin du dixième et dernier épisode de la première saison. L'action, difficilement perceptible, se passe de nuit. Elle est commentée pour le bureau parisien par la voix basse, très étouffée, d'un officier présent sur les lieux. Les cadrages choisis, vues d'ensemble et plans moyen, sont de l'ordre de la retenue. Si l'action est rapide, la représentation est radicalement différente de celle propre à 24 heures chrono par exemple, assénée dans un montage dynamique fait d'une accumulation de plans extrêmement courts alternant gros plans, plans rapprochés et plans d'ensemble dans un rythme saccadé souligné par une partition musicale appuyée. Ici, il y a peu d'action, ce qui se passe reste opaque aux yeux du spectateur comme à ceux des personnages. Les situations décrites sont parfois éprouvantes (les scènes d'interrogatoire inopiné en vue de préparer la nouvelle recrue), parfois ennuyeuses (une vie de bureau sans grand relief pour des personnages anodins et sans grande passion). Le récit tient sur une écriture blanche et un jeu des acteurs très en retrait qui étaient aussi la marque du film de Rochant consacré à l'espionnage. Le domaine du renseignement figuré est un monde très calme, sans hystérie ni énervement, les voix sont posées, douces même à l'image de Malotru (incarné par Mathieu Kassovitz), charmant mais évasif, dégageant une impression de neutralité professionnelle. Jouant de ses multiples identités, il est toujours sur son quant-à-soi. Les dimensions héroïques 
(Malotru se fait tabasser sans réagir par un agent/garde du corps syrien afin de ne pas lever le doute sur sa mission) et sexy évacuées, il reste une vie de bureau.

\section{Le quotidien du renseignement}

\section{La construction d'un individu dépendant, éthos de l'espion}

La première saison de la série décrit en détail le processus de fabrication de l'identité de substitution de plusieurs clandestins, ainsi que le processus d'effacement de cette identité. Le candidat au départ répète son rôle face au veilleur. Studieusement, la jeune recrue choisie pour l'Iran améliore son persan, révise ses connaissances techniques en géophysique, répète son rôle, apprend les techniques de repérage sur son environnement. L'apprentissage est intense, les tests impromptus (cinq minutes pour découvrir l'identité d'inconnus dans un restaurant) ou camouflés (arrestation injustifiée et interrogatoire musclé). La surveillance du futur ou ex-clandestin est centrale : un des procédés retenus pour rendre compte de cette surveillance est celui de l'écran dans l'écran. Certaines séquences sont vues à travers l'écran d'un ordinateur (image granuleuse en noir et blanc), laissant supposer l'enregistrement par le biais de caméras de surveillance à l'insu ou au vu des personnages.

L'entraînement qui laisse peu de temps à soi n'est qu'un des aspects de la prise en charge du clandestin, l'infantilisation en est un autre. Tout est pensé pour lui ou pour elle, à sa place. Les clés de l'appartement, de la voiture, etc. lui sont tendues de même que la couverture semble avoir été définie par l'équipe du bureau sans que le clandestin n'ait son mot à dire. L'emploi du temps est planifié pour lui/elle. Cette absence d'initiative contraste avec ce que l'on imagine être le travail de terrain. L'ensemble du processus semble avoir pour objet de gommer les aspérités de la personnalité du candidat, de limiter sa capacité à prendre des initiatives pour le conformer au comportement attendu (le plus neutre possible, le plus attentiste en apparence) où les réflexes inculqués auront plus d'importance que l'autonomie. La clandestine en partance pour Téhéran est sortie de l'avion à Tbilissi, détenue indéfiniment dans un hôtel sans explication. Quand la panique commence à monter, son réflexe est de contacter celui qui la sortira de ce mauvais pas. Et quand elle parvient à téléphoner, c'est son patron iranien (donc celui de son identité de légende) et non son patron de la DGSE qu'elle appelle, préservant ainsi sa crédibilité et sa mission.

Dernier élément qui n'est pas spécifique à cette série, l'espion s'inscrit dans un contexte organisationnel à la dimension familiale très marquée. Ici, ce registre de l'intime apparaît dans les dénominations : les agents chargé de Malotru à son retour en France sont Pépé et Mémé. Dans la sixième saison de Chapeau melon et bottes de cuir, le supérieur hiérarchique de John Steed est Mère-Grand (Mother pour la version anglaise). Dans les James Bond, l'initiale M tient pour le nom du directeur du MI6 qu'il soit incarné par une femme ou un homme - faut-il y voir une mère pour ses agents? London Spy va plus loin en faisant des parents de l'agent du MI6 éliminé des espions et attribuant à sa mère un rôle déterminant dans la tentative de récupération de l'agent aboutissant à la mise en scène macabre de sa mort. Certains membres de l'équipe du Bureau des légendes s'invitent dans l'intimité des clandestins : surprenant la fille de Malotru lors d'une visite de contrôle ou débarquant chez la jeune recrue avant son départ pour l'Iran avec ses mots « Avec qui tu pourrais faire la fête ?» L'organisation devient une famille de substitution avec ses non-dits, ses tensions, ses routines et plaisanteries (les noms des clandestins correspondent aux insultes favorites du capitaine Haddock). Le fonctionnement de la mafia nous a appris que le fait de faire reposer l'organisation sur la structure familiale permet d'en maintenir le strict contrôle et de s'assurer de l'adhésion et du silence des membres de la famille sur l'activité secrète qui les occupe. 


\section{Un certain génie bureaucratique}

Le travail montré au quotidien au sein du bureau des légendes est celui d'une organisation bureaucratique cloisonnée. Dans une atmosphère vieillotte (le décorateur a privilégié les tons beige et bruns), s'observe une activité des bureaux qui à première vue semble habituelle. Ce qui frappe le premier regard est l'encombrement de lieux étriqués et peu modernes. Les jeux d'acteurs en interne vont de l'auto-justification au camouflage des déficiences. Leurs stratégies dans un système hiérarchisé n'ont rien de spécifique. Les uns affirment leur position - quand trois directeurs discutent d'une opération à annuler dans la salle de sport de l'immeuble, ils congédient un jeune fonctionnaire par un définitif « c'est l'heure des directeurs $»-$, les autres tentent de préserver un degré d'autonomie par rapport à la ligne hiérarchique et aux métiers : nouveaux arrivés dans les bureaux, ils manœuvrent à vue, qu'il s'agisse de la psychologue comportementaliste ou de l'ex-clandestin Malotru.

La gestion des situations de crise paraît tenir de l'exceptionnel et implique de travailler avec d'autres équipes au sein d'une cellule de crise : ainsi le règlement de l'enlèvement du clandestin par l'organisation Etat islamique au Sahel suscite une envolée d'applaudissements de la part des équipes concernées à Paris. De même, la résolution du problème de la jeune clandestine disparue à Tbilissi voit l'équipe célébrer avec champagne et applaudissements. Ainsi la conclusion de la première saison signe la réussite du retour de Malotru au sein de son service et laisse imaginer une carrière prometteuse sur laquelle d'aucuns ont déjà parié.

\section{Retour sur l'objet de l'enquête}

En évacuant une grande partie de l'imagerie associée à l'espionnage, en n'ayant pas peur du temps long et de l'ennui, cette série parvient à instaurer une représentation inédite dans le paysage télévisuel. Le récit se concentre sur une activité plus laborieuse que romanesque. Mais alors, comment valoriser ce que le sens commun juge stérile ? La question se pose dans la fiction à propos de la mission de renseignement, très éloignée de la représentation commune et dépourvue des éléments glamour propres aux James Bond. Résumée par un fonctionnaire dans la série d'Eric Rochant: "Votre mission, c'est connaître les gens sans vous faire remarquer ». Pas d'engagement dans l'action, pas un travail d'analyste, une bureaucratie discrète, qui ne fait pas rêver et laisse même dubitatif. Ainsi, l'agent de renseignement face à sa fille adolescente s'explique à son retour : " ma mission c'était de lier connaissance » et rien de ce que la famille du clandestin pouvait imaginer : «C'est pour ça que t'es parti six ans, pour te faire des connaissances?»

\section{REFERENCES BIBLIOGRAPHIQUES}

BoltansKi, L., Énigmes et complots. Une enquête à propos d'enquêtes, Paris, Gallimard, coll. « NRF essais », 2012.

Bonin, H., «James Bond, de la littérature populiste au film populaire : l'angoisse de la conspiration et du sur-pouvoir capitalistes », Paris, BNF, colloque James Bond, 17 janv. 2007. Bulinge, F., De l'espionnage au renseignement, Paris, Vuibert, 2012.

GREY, C., "An Organizational Culture of Secrecy: the Case of Bletchley Park», Management and Organizational History, vol. 9, n 1, fév. 2014, p. 107-122. 
Plancade, J.-P., Production audiovisuelle : pour une politique industrielle au service de l'exception culturelle, rapport d'information $\mathrm{n}^{\circ} 616$ (2012-2013) fait au nom de la commission de la culture, de l'éducation et de la communication du Sénat, 30 mai 2013. 